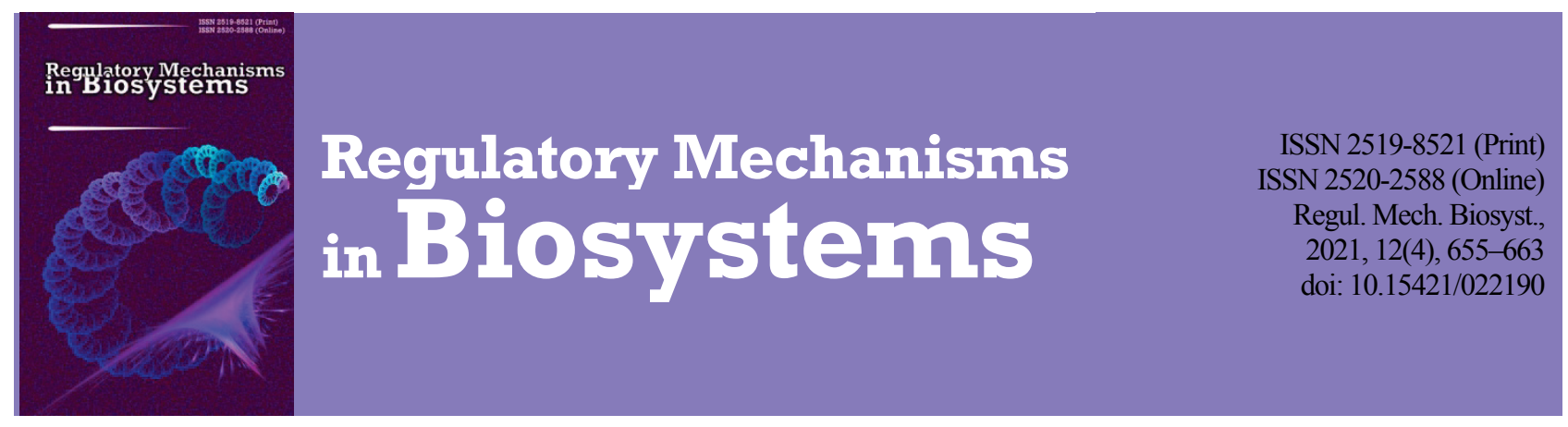

\title{
Elimination of the toxic effect of copper sulfate is accompanied by the normalization of liver function in fibrosis
}

\author{
A. I. Bozhkov, A. A. Bozhkov, I. E. Ponomarenko, N. I. Kurguzova, \\ R. A. Akzhyhitov, A. V. Goltvyanskii, E. M. Klimova, S. O. Shapovalov
}

V. N. Karazin Kharkov National University, Kharkov, Ukraine

Article info

Received 25.09.2021

Received in revised form 18.10.2021

Accepted 20.10.2021

V.N. Karazin Kharkov National University, Pl. Svobody, 4,

Kharkov, Ukraine

Tel.: +38050-402-36-21.

E-mail:niibio@karazin.ua

Rivne State University of Humanities,

Stepana Bandery St., 12 Rivne, 33028, Ukraine.

Tel. +38-098-259-37-57. E-mail:

irchukmail@gmail.com
Bozhkov, A. I., Bozhkov, A. A., Ponomarenko, I. E., Kurguzova, N. I., Akzhyhitov, R. A., Goltvyanskï, A. V., Klimova, E. M., \& Shapovalov, S. $O$. (2021). Elimination of the toxic effect of copper sulfate is accompanied by the normalization of liver function in fibrosis. Regulatory Mechanisms in Biosystems, 12(4), 655-663. doi:10.15421/022190

The search for biologically active compounds that regulate liver function in fibrosis is an urgent medical and biological problem. A working hypothesis was tested, according to which low molecular weight biologically active compounds from Pleurotus ostreatus and Sacharamirses cerevisiae are capable of exerting immunomodulatory and antitoxic effects after intoxication of the body with ions of heavy metals, in particular copper sulfate. Elimination of the toxic effect caused by copper sulfate can also ensure the normalization of liver function in various pathologies, in particular with liver fibrosis. When determining toxicity, a study was carried out on Wistar rats, and when studying the effect of low molecular weight biologically active compounds on liver function, clinical trials were carried out on volunteers. The activity of alanine aminotransferase, aspartate aminotransferase, actonitase and glutathione peroxidase, as well as the content of bilirubin and lipid hydroperoxides were determined. It was shown that preliminary administration of biologically active compounds to rats at a dose of $0.05 \mathrm{~mL} / 100 \mathrm{~g}$ of body weight provided the formation in some animals (up to $80 \%$ ) of resistance to the toxic effect of copper sulfate (dose $2.5 \mathrm{mg} / 100 \mathrm{~g}$ of body weight). Such stability is associated with a shift in the balance of "prooxidantsantioxidants" towards antioxidants. The data obtained in the clinic on volunteers with liver fibrosis and hepatitis also testify in favour of the membranotropic action of biologically active compounds. Biologically active compounds provided a decrease or complete restoration of the activity of transferases (ALT and AST) in the blood serum of these patients, with the exception of one patient out of 20 examined. Our experiment has shown the relationship between the elimination of toxicity to the action of copper sulfate and the normalization of liver function in patients. The results obtained indicate that it will be promising to use a complex of low molecular weight components from $P$. ostreatus and S. cerevisiae as an antidote and hepatoprotective agent.

Keywords: toxicity of copper sulfate; transferases; lipid hydroperoxides; aconitase; glutathione peroxidase; mitochondria; erythrocytes.

\section{Introduction}

As is well-known, the liver plays a leading role in the formation of the body's adaptive responses to the action of various environmental factors. Transformation, detoxification, and elimination of xenobiotics from the body are carried out due to the functional activity of the liver (Williams \& Iatropoulos, 2002; Parkinson \& Ogilvie, 2008; Piven et al., 2020; Slivinska et al., 2021). Excessive load on the functional activity of the liver is accompanied by the formation of liver pathologies, which manifests itself as hepatitis, fibrosis, cirrhosis of the liver (Puche et al., 2011; JaramilloJuárez et al., 2008), which may be accompanied by the formation of comorbid states (Poordad, 2015).

Pathogenetic factors of the liver are classified into biogenic (viruses, microorganisms), physical (radiation, electromagnetic fields), chemical (heavy metal ions and a wide range of hepatotropic poisons); drugs, and even some types of food and alcoholic beverages (Lynes et al., 2007; Gao \& Bataller, 2011; Satapathy et al., 2015; Hirsova et al., 2016).

In this regard, the liver can be considered as a metabolic mediator between environmental factors and the body, in the sense that it transforms "the actions of environmental factors into metabolic reactions of the body", while actively rebuilding its metabolism, which is quite often accompanied by the formation of chronic pathologies - fibrosis and cirrhosis of the liver. In 2017, 1.32 million people died from irreversible liver pathology (Buchanan \& Sinclair, 2020), chronic liver pathologies and cirrhosis were diagnosed in $1.8 \%$ ( 4.5 million people) of the US population and are included in the list of 10 most common causes of death in developed countries (Sepanlou et al., 2017). The development of "protection" and treatment of liver diseases is an urgent biomedical and social problem since the number of people suffering from various liver pathologies in the world is even more than documented.

Currently, the main approaches to solving this problem are reduced to: 1 - elimination of negative hepatotropic factors (Francavilla et al., 1994; Myers et al., 2002); 2 - development of means for regulating liver function, in particular, hepatoprotectors; 3 - development of regenerative medicine - and, first of all, cell therapy (Mason \& Manzotti, 2010; Bozhkov et al., 2014); 4 - improving the methods of liver transplantation (Rana \& Godfrey, 2019).

Currently, a huge number of hepatoprotective drugs have been developed (Verma et al., 2013; Saito et al., 2016; Shirani et al., 2017; Xu et al., 2018; Owojuyigbe et al., 2020). However, their effectiveness, especially to fibrosis, cirrhosis, and oncological processes in the liver, is rather low, and in most cases, they have side effects on the body (Shirani et al., 2017; Fahmy et al., 2020).

In this regard, it is necessary to search for new natural means of regulating liver functions. As in this case, along with various functions, the liver is capable of regeneration (Francavilla et al., 1994; Michalopoulos \& DeFrances, 1997; Gagliano et al., 2007). The induction of regenerative processes in the liver involves a variety of signaling molecules, including such nonspecific components as "products" of cell hydrolysis. It has been suggested that low molecular weight components from various biological 
sources, which are formed as a result of molecular hydrolysis, may have a hepatoprotective effect (Bozhkov et al., 2017).

We believe that the main problem in the regulation of liver function, in particular in fibrosis, can be solved by the development of new substances of biological origin.

To test this hypothesis, we obtained a biologically active substance, which includes low molecular weight proteins and peptides (with a molecular weight of no more than $30 \mathrm{kDa})-21.0 \%$, oligosaccharides $-67.0 \%$, lipids $-11.0 \%$, vitamins, and minerals $-1.0 \%$, then there is a wide range of biologically active components of the Pleurotus ostreatus and Sacharamirses cerevisiae fungi (Kurguzova et al., 2015), and its hepatoprotective action was assessed experimentally and clinically.

Previous studies have shown that biologically active components from $P$. ostreatus and $S$. cerevisiae can eliminate acute infectious diarrhea (colibacillosis) in experimental animals (calves, piglets), modulate the immune system, reduce the manifestation of oxidative stress, and normalize liver function in the case of induced liver fibrosis in animals, increase the performance of animals in the test swimming with a load, against the background of toxic poisoning with copper ions (Bozhkov et al., 2016).

Since metal ions, and in particular copper ions, are inducers of the development of liver fibrosis (Lakherwal, 2014), in this work we determined the ability of biologically active components from $P$. ostreatus and $S$. cerevisiae to prevent the death of experimental animals (Wistar rats), in acute poisoning with copper sulfate, some indicators of the redox system of the cell (activity of aconitase, glutathione peroxidase and the content of lipid hydroperoxides in liver mitochondria), which change with liver fibrosis. In liver pathologies some of the hepatocytes are destroyed, which is accompanied by the transition of several ALT and AST transferases from hepatocytes into the bloodstream (which is most often used in the diagnosis of liver pathology). Therefore, the activity of alanine aminotransferase (ALT), aspartate aminotransferase (AST), as well as bilirubin, as an index of the product of hemoglobin hydrolysis in patients with hepatitis and liver fibrosis was also estimated.

\section{Materials and methods}

Experiments on laboratory animals, including assessing the effect of copper sulfate, were carried out in agreement with the bioethical committee of V. N. Karazin, which is guided by the provisions of the "European Convention for the Protection of Vertebrate Animals used for Experimental and other Scientific Purposes" (Strasbourg, March 18, 1986). The animals received thiopental anesthesia, after which decapitation and dissection of the animals were performed. Biologically active components of the fungi $P$. ostreatus and $S$. cerevisiae are approved for use and before use, all patients were informed about clinical studies and gave their consent to participate in the study; these studies were carried out in the clinic in compliance with accepted standards ("WMA declaration of Helsinki", 2013).

Experiments on the effect of biologically active compounds from $P$. ostreatus and $S$. cerevisiae on resistance to the toxic effect of copper sulfate were carried out on young, sexually mature male Wistar rats (3.0 3.5 months), weighing $150-200 \mathrm{~g}$. In a group of 75 rats $(n=15), 5$ experimental subgroups were formed: I - control received daily per os saline, in a volume of $0.05 \mathrm{~mL} / 100 \mathrm{~g}$ of body weight, for 6 days; II - received biologically active compounds from $P$. ostreatus and $S$. cerevisiae daily at a dose of $0.01 \mathrm{~mL} / 100 \mathrm{~g}$ of body weight, which was injected with per os, for 6 days; III - received biologically active compounds from $P$. ostreatus and $S$. cerevisiae daily at a dose of $0.05 \mathrm{~mL} / 100 \mathrm{~g}$ of body weight which was injected with per os, for 6 days; IV - received biologically active compounds from $P$. ostreatus and $S$. cerevisiae daily at a dose of $0.4 \mathrm{~mL} / 100 \mathrm{~g}$ of body weight which was injected with per os, for 6 days; $\mathrm{V}$ - received biologically active compounds from $P$. ostreatus and S. cerevisiae daily at a dose of $0.8 \mathrm{~mL} / 100 \mathrm{~g}$ of body weight which was injected with per os, for 6 days. On the 7 day, all animals were injected with copper sulfate at a dose of $2.5 \mathrm{mg} / 100 \mathrm{~g}$ of body weight. All manipulations with animals (the administration of biologically active compounds from $P$. ostreatus and $S$. cerevisiae, determination of body weight and body temperature), were carried out from 9:00 a.m. to 11:00 a.m. before feeding the animals. All animals were kept in standard vivarium condi- tions at a temperature of $18-20^{\circ} \mathrm{C}$, a natural change in the lighting regime, and had free access to food and water.

When determining the effect of biologically active compounds from $P$. ostreatus and $S$. cerevisiae on the indicators of the redox system, a group of 3-month-old rats of 15 animals was formed, which were divided into 3 subgroups of 5 animals each: I - group was not exposed to any influence; II - liver fibrosis was induced by three times administration of copper sulfate with an interval between administration of 48 hours at a dose of $1 \mathrm{mg} / 100 \mathrm{~g}$ of body weight, as described previously (Bozhkov et al., 2010), and 24 hours after administration at the last administration, the animals were decapitated after ether anesthesia; III - liver fibrosis was induced according to the same scheme, followed by per os administration of biologically active compounds from $P$. ostreatus and $S$. cerevisiae to these animals at a dose of $0.05 \mathrm{~mL} / 100 \mathrm{~g}$ for three days, and 24 hours after the last administration of biologically active compounds from $P$. ostreatus and $S$. cerevisiae, the animals were decapitated after ether anesthesia.

When the animals were decapitated, blood was collected, blood serum was obtained. Mitochondria were isolated from the liver (Kamath \& Narayan, 1972), and the content of lipid hydroperoxides, mitochondrial aconitase activity and glutathione peroxidase was determined.

The mitochondria swelling was recorded by changing the optical density in a thermostatted $\left(37^{\circ} \mathrm{C}\right)$ cuvette with constant agitation by spectrophotometer Specord UV VIS (Germany) at $610 \mathrm{~nm}$. The incubation medium composition was the following: $10 \mathrm{mM}$ Tris- $\mathrm{HCl}, \mathrm{pH} 7.4$,

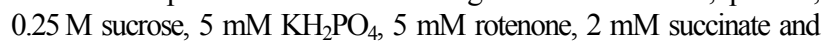
$25 \mathrm{mM} \mathrm{CaSO}_{4}$. The lipid hydroperoxide (HPL) content of mitochondria was determined by method (Asakawa \& Matsushita, 1980). The HPL content in serum was determined as previously described. The absorption spectrum of the coloured product was recorded on double-beam spectrophotometer Specord UV VIS, measuring the difference in extinction at 535 and $520 \mathrm{~nm}$. The HPL content was expressed in equivalent amounts of using a molar extinction coefficient of $1.56 \times 10^{5} \mathrm{M}^{-1} \mathrm{~cm}^{-1}$. The activity was expressed in nmol $\mathrm{MDA} / \mathrm{mg}$ protein.

The activity rate of aconitate hydratase (aconitase, EC 4.2.1.3) was measured according to the procedure described in (Varghese et al., 2003) and expressed in nmol of aconitate per $1 \mathrm{mg}$ of proteins using the molar extinction coefficient $3.6 \mathrm{mM}^{-1} \mathrm{~cm}^{-1}$.

The activity rate of glutathione peroxidase (EC 1.11.1.9, GP) was measured in liver mitochondria by spectrophotometry according to the method described in (Paglia \& Valentine, 1967): we added $50 \mathrm{mM} \mathrm{K} / \mathrm{Na}$ phosphate buffer ( $\mathrm{pH} 7.4$ ) containing $1 \mathrm{mM}$ of DTA, $0.15 \mathrm{mM}$ of NADPH, 1 unit of yeast glutathione reductase, $0.2 \%$ triton X-100, and $3 \mathrm{mM}$ sodium azide for inhibiting catalase. The activity rate was expressed in nmol NADPH/min per $1 \mathrm{mg}$ of proteins or $1 \mathrm{~mL}$ of serum with allowance for the molar extinction coefficient $6.22 \times 10^{3} \mathrm{M}^{-1} \mathrm{~cm}^{-1}$.

Assessment of the hepatoprotective effect of biologically active components of the fungi $P$. ostreatus and $S$. cerevisiae. To assess the hepatoprotective effect of components of the fungi, in the clinic where patients with liver fibrosis and hepatitis are treated, a group of 20 volunteers was formed who took biologically active compounds daily 30 minutes before meals at a dose of $0.01 \mathrm{~mL}$ per kilogram of body weight for 10 days. Before taking biologically active compounds from $P$. ostreatus and $S$. cerevisiae, all volunteers had their blood biochemical parameters determined: the activity of ALT, AST, and the content of bilirubin (by conventional methods using standard kits Stat Fax 1904). After 10 days, the biochemical activities of ALT, AST, and the bilirubin content were redetermined in all patients. The patients were represented by 9 men and 11 women, aged 40-60 years. The study involved patients with a wide range of liver pathologies (clinical picture of patients: acute toxic infections; use of high doses of antibiotics (pneumonia); chronic hepatitis (including autoimmune); fatty degeneration; toxic alcoholic hepatosis, but in all subjects, the activity of ALT and ATC in the blood serum was increased.

The data are presented in the figures as $\mathrm{x} \pm \mathrm{SE}$ ( $\mathrm{x} \pm$ standard error). The statistical analysis of survival experimental animals was carried out by the moment method (Kaplan-Meier with log-rank test with Yates' correction); the estimate of the accuracy of the approximation was calculated using the Greenwood formula. Comparison of the experimental and control groups was carried out using a log-rank criterion. The results obtained 
were presented for each individual, as well as their group mean values (in laboratory animals only the average for the group), which were subjected to statistical processing using analysis of variance, where the differences were considered significant at $\mathrm{P}<0.05$ (taking into account the Bonferroni amendment).

\section{Results}

It turned out that if the animals (15 sexually mature healthy males) were injected with copper sulfate solution at a dose of $2.5 \mathrm{mg} / 100 \mathrm{~g}$ of body weight, then after 2-3 hours from the moment of administration, up to $60.0 \%$ of the animals died (median equal to 3 ), and after 20 hours from

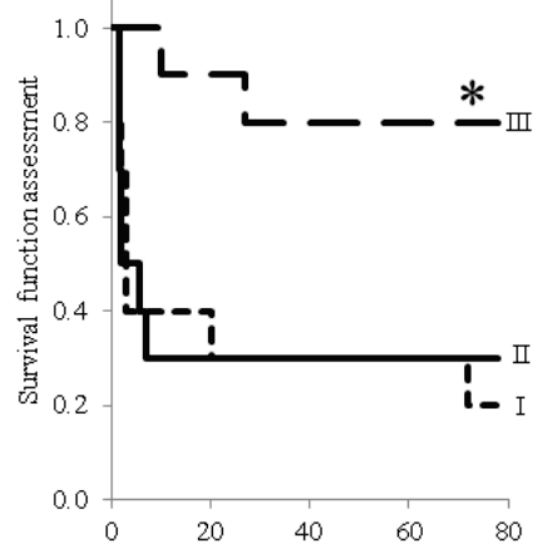

the beginning of the experiment in the control group $30.0 \%$ of the animals remained alive. By the third day of the experiment, only $20.0 \%$ of the animals remained alive (Fig. 1a, curve I). Consequently, copper sulfate at a dose of $2.5 \mathrm{mg} / 100 \mathrm{~g}$ of body weight exhibits acute toxicity for Wistar rats.

In the event that the animals received daily per os biologically active compounds from $P$. ostreatus and $S$. cerevisiae, which is represented by a complex of low molecular weight proteins (Fig. 2), at a dose of $0.01 \mathrm{~mL} / 100 \mathrm{~g}$ of body weight for 6 days, and 24 hours after the last administration, they were injected with copper sulfate at a dose of $2.5 \mathrm{mg} / 100 \mathrm{~g}$, as well as the control group, the mortality curve did not differ significantly from the control option (Fig. 1). $b$

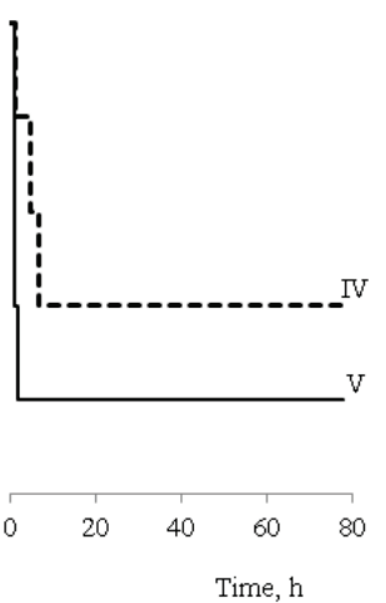

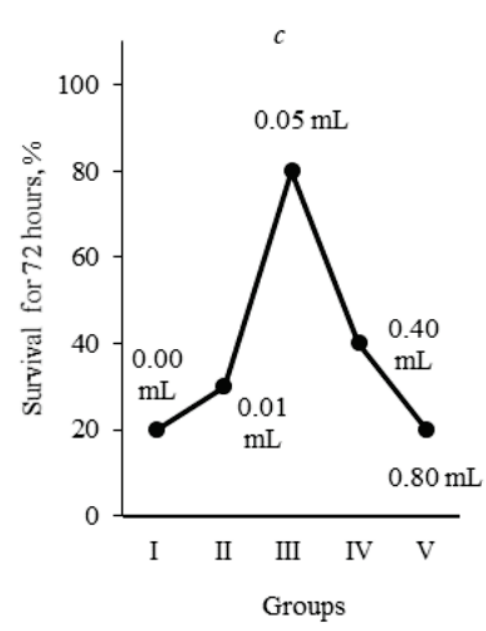

Fig. 1. Survival of experimental animals according to Kaplan-Meier $(\mathrm{N}=75)$ : $a$-control group, which did not receive biologically active compounds from $P$. ostreatus and $S$. cerevisiae, they were injected with copper sulfate at a dose of $2.5 \mathrm{mg} / 100 \mathrm{~g}$ of body weight (I); animals that were injected daily with compounds at a dose of $0.01 \mathrm{~mL} / 100 \mathrm{~g}$ of body weight for 6 days, followed by the administration of copper sulfate at a dose of $2.5 \mathrm{mg} / 100 \mathrm{~g}$ (II); a group of animals that were injected daily with compounds at a dose of $0.05 \mathrm{~mL} / 100 \mathrm{~g}$ of body weight for 6 days, followed by the administration of copper sulfate at a dose of $2.5 \mathrm{mg} / 100 \mathrm{~g}$ (III); $b$ - a group of animals that were previously injected with compounds at a dose of $0.4 \mathrm{~mL} / 100 \mathrm{~g}$ of body weight according to the same scheme, followed by the administration of copper sulfate (IV) and a group that was previously injected with compounds at a dose of $0.8 \mathrm{~mL} / 100 \mathrm{~g}$ with the subsequent administration of copper sulfate $(\mathrm{V}) ; c$ - dose dependence of the survival of rats in arbitrary units, 72 hours after administration of copper sulfate to animals at a dose of $2.5 \mathrm{mg} / 100 \mathrm{~g}$ of body weight; ${ }^{*}$ - significant values are marked $(\mathrm{P}<0.05)$ compared to the intact level (log-rank test with Yates' correction)

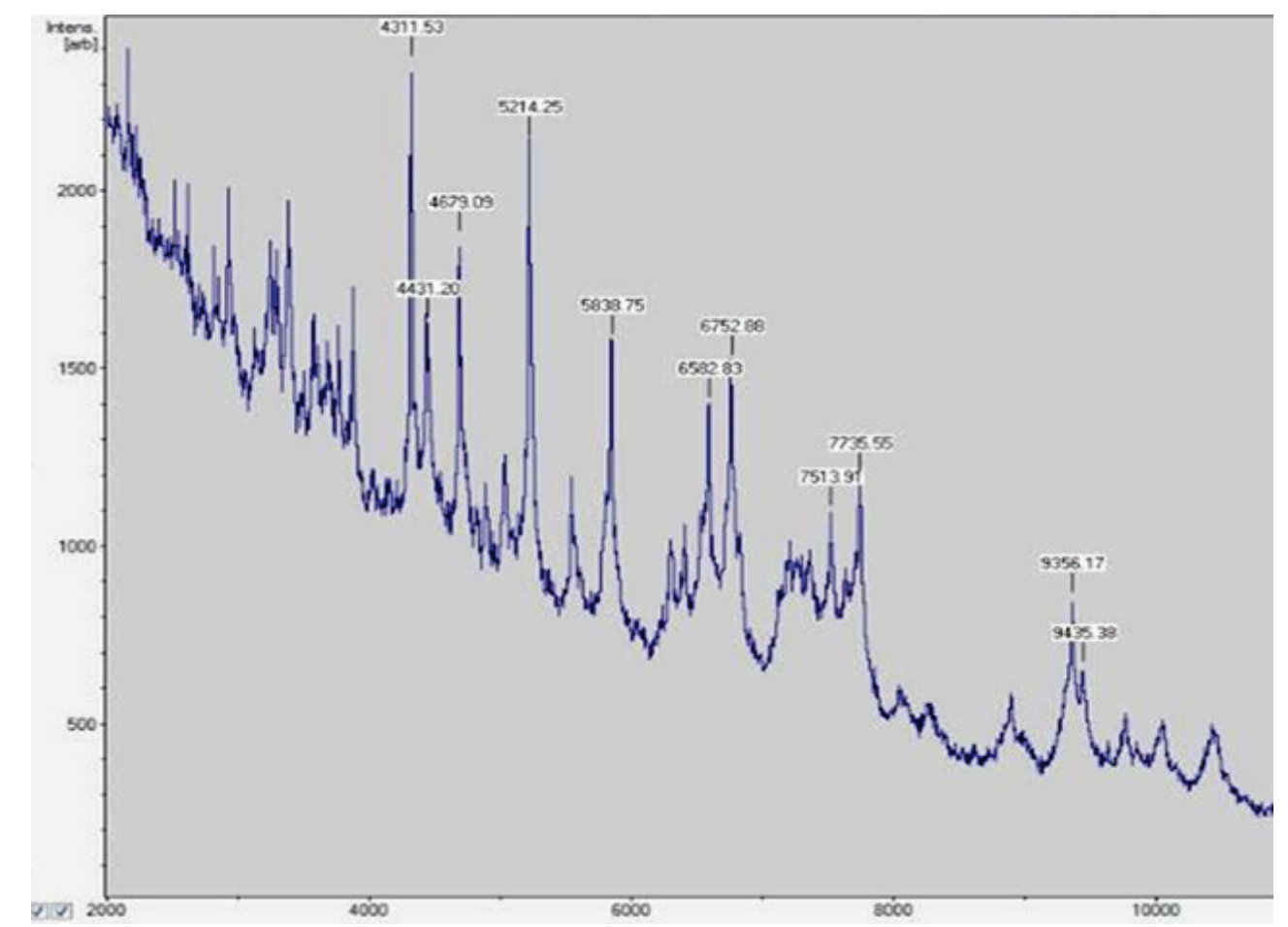

Fig. 2. A typical spectrum of proteins that make up biologically active compounds from P. ostreatus and S. cerevisiae were obtained on a Microflex MALDI-TOF Biotyper mass spectrometer (Bruker): the ordinate is relative units, the abscissa is $\mathrm{m} / \mathrm{z}$ of proteins 
If the animals were preliminarily injected with biologically active compounds from $P$. ostreatus and $S$. cerevisiae for 6 days at a dose of $0.05 \mathrm{~mL} / 100 \mathrm{~g}$ of body weight, then a reliable manifestation of resistance to the lethal effect of copper sulfate was observed. So, the death of the first animals after the administration of copper sulfate occurred much later (only after 10 hours), compared with the control group, and for 20 hours $90.0 \%$ of the animals remained alive, and after three days $80.0 \%$ of the animals were active and did not differ from normal animals in behaviour (Fig. 1a, curve III).

An increase in the biologically active compounds from $P$. ostreatus and $S$. cerevisiae dose to $0.4 \mathrm{~mL} / 100 \mathrm{~g}$ of body weight did not significantly affect the resistance of experimental animals to the toxic effect of copper sulfate, as did the dose of $0.8 \mathrm{~mL} / 100 \mathrm{~g}$ (Fig. 1b, curves IV and V). It should be noted that the components of biologically active compounds, even at a dose of $0.8 \mathrm{~mL} / 100 \mathrm{~g}$ of mass, did not show any negative toxic effects on the body. Consequently, preliminary administration of biologi-
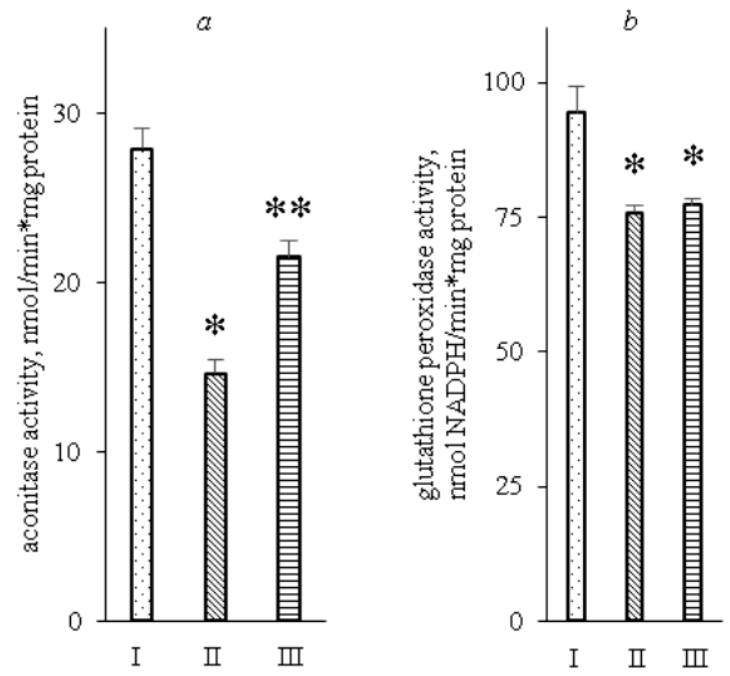

cally active compounds from $P$. ostreatus and $S$. cerevisiae to experimental animals increased their resistance to the toxic effect of copper sulfate, and this effect depended on the dose and had a U-shaped character. The greatest protection effect was observed for a dose of $0.05 \mathrm{~mL} / 100 \mathrm{~g}$ of body weight (Fig. 1c).

In the next series of experiments, the aconitase activity, one of the enzymes of the Krebs cycle in animals with Cu-induced liver fibrosis, was determined. It was found that after 3 consecutive injections of copper sulfate in experimental animals, at doses of $1 \mathrm{mg} / 100 \mathrm{~g}$ of body weight, which was about $30.0 \%$ of the lethal dose, and induced the development of liver fibrosis (Bozhkov et al., 2010), the aconitase activity in mitochondria was reduced by $48.0 \%$ compared with a control level (Fig. 3a). If animals with liver fibrosis previously received biologically active compounds from $P$. ostreatus and S. cerevisiae at a dose of $0.05 \mathrm{~mL} / 100 \mathrm{~g}$ for three days, the aconitase activity was lower than the control level by only $23.0 \%$ and significantly higher than after administration of copper sulfate (Fig. 3a).
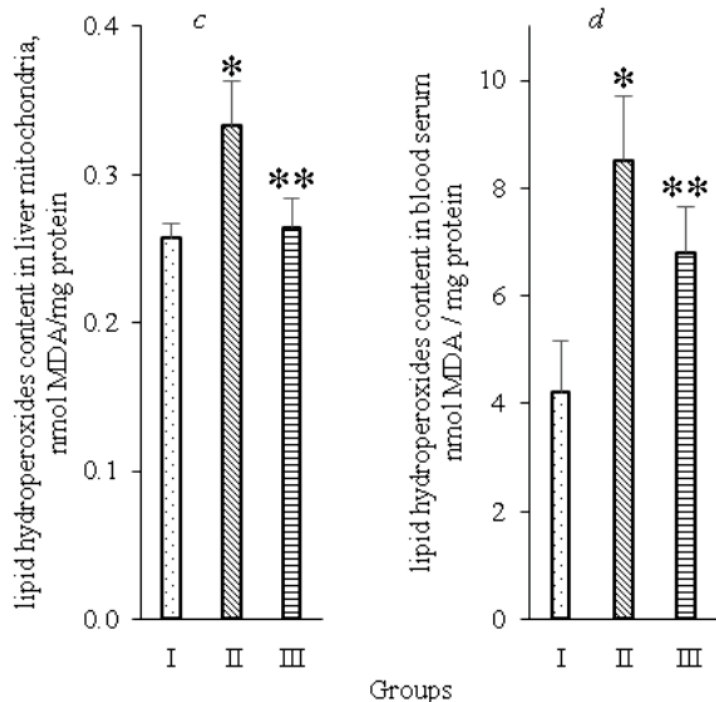

Fig. 3. The activity of aconitase and glutathione peroxidase, as well as the content of lipid hydroperoxides in experimental groups of animals $(\mathrm{x} \pm \mathrm{SE}, \mathrm{N}=15): a$ - the aconitase activity in liver mitochondria in intact animals (I); in animals with Cu-induced liver fibrosis (II) and in animals with $\mathrm{Cu}$-induced liver fibrosis, which were injected with biologically active compounds from $P$. ostreatus and $S$. cerevisiae per os at a dose of $0.05 \mathrm{~mL} / 100 \mathrm{~g}$ of body weight for 3 days (III); $b$ - activity of glutathione peroxidase in liver mitochondria in the same experimental groups; $c$-the content of lipid hydroperoxides in liver mitochondria in the same groups of animals; $d$ - the content of lipid hydroperoxides in blood serum in the same groups of animals; the mean values, standard error are presented, ${ }^{*}$ - significant values $(\mathrm{P}<0.05$, taking into account the Bonferroni amendment) compared with the intact level and ** - compared with Cu-induced liver fibrosis after biologically active compounds from $P$. ostreatus and $S$. cerevisiae administration are noted

It is known that a decrease in aconitase activity correlates with an increase in lipid hydroperoxides in mitochondria; therefore, it was of interest to determine the activity of the key enzyme of antioxidant defense, glutathione peroxidase, in liver mitochondria. And it was found that the activity of glutathione peroxidase in the mitochondria of the liver of rats which were injected three times with copper sulfate, was reduced by $20.0 \%$ compared with the control values. At the same time, administration of biologically active compounds from $P$. ostreatus and $S$. cerevisiae to experimental animals with liver fibrosis did not significantly change the activity of this enzyme (Fig. 3b).

Determination of the content of lipid hydroperoxides in mitochondria in animals with liver fibrosis showed that their content was increased by $29.0 \%$ compared to the control level (Fig. 3c). Administration of biologically active compounds from $P$. ostreatus and $S$. cerevisiae to animals with liver fibrosis reduced the content of lipid hydroperoxides in mitochondria by $29.0 \%$ and their content did not differ from the values of control animals (Fig. 3c).

Even more, pronounced changes in the content of lipid hydroperoxides were observed in blood serum. So, after the administration of copper sulfate, their content in it increased by $102.0 \%$ compared to the control, and after the administration of biologically active compounds from $P$. ostreatus and S. cerevisiae, their content decreased by $20.0 \%$ and exceeded the control by $60.0 \%$ (Fig. 3d). The results obtained allow us to conclude that biologically active compounds from $P$. ostreatus and $S$. cerevisiae have a "protective" effect against the toxic effect of copper sulfate, which can be realized at the level of regulation of the body's redox system parameters. This is supported by the previously obtained data on the presence of antioxidant and antiradiation activity of biologically active compounds from $P$. ostreatus and $S$. cerevisiae in the in vitro.

However, the protective effect of the low molecular weight substance biologically active compounds from $P$. ostreatus and $S$. cerevisiae cannot be provided only at the level of the redox system. It was found that indeed the number of erythrocytes in animals that received copper sulfate was reduced in comparison with the control (Fig. 4). The administration of biologically active compounds to animals with liver fibrosis ensured the preservation of their quantity, at the level of control values (Fig. 4).

Consequently, biologically active compounds from $P$. ostreatus and S. cerevisiae can also exhibit membranotropic action. In this regard, it can be expected that such a membranotropic effect will be manifested in hepatocytes. Considering that biologically active compounds from $P$. ostreatus and $S$. cerevisiae are obtained from substrates used in food, without the use of toxic chemicals, it does not have any negative effects, eliminates some changes in liver fibrosis, and has a protective effect at a dose of $0.05 \mathrm{~mL} / 100 \mathrm{~g}$ of body weight. It was of interest to study its effect on patients with hepatitis and liver fibrosis in the clinic.

In a clinical hospital, a group of 20 patients, men (9) and women (11), aged 40-60 years, was formed, suffering from liver pathologies, which are accompanied by an increase in the activity of ALT, AST, and the content of bilirubin (Fig. 5-7). 


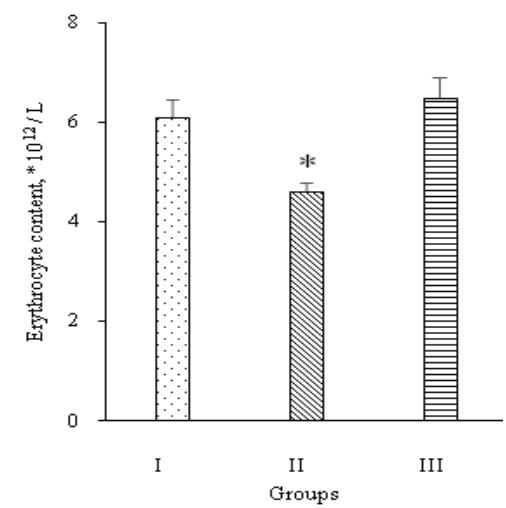

Fig. 4. Content of erythrocytes ( $x \pm S E, N=15)$ : the number of erythrocytes in the blood of intact animals (I); animals that were injected with copper sulfate at a dose of $1 \mathrm{mg} / 100 \mathrm{~g}$ of body weight three times with an interval between injections of 48 hours (II); animals that received biologically active compounds from $P$. ostreatus and $S$. cerevisiae at a dose of $0.05 \mathrm{~mL} / 100 \mathrm{~g}$ of body weight, followed by the administration of copper sulfate at a dose of $1 \mathrm{mg} / 100 \mathrm{~g}$ of body weight (III); * - significant values are noted $(\mathrm{P}<0.05)$ compared to the intact level (taking into account the Bonferroni amendment)

Before the start of intake of biologically active compounds from $P$. ostreatus and $S$. cerevisiae, the serum ALT activity in all examined patients was increased: from $26.5 \%$ compared with the upper limit of reference values in 5 patients, up to 2 times in 15 patients. On average, in all patients, the activity of this marker enzyme was $86.5 \%$ higher than normal (Fig. 5a). A ten-day course of biologically active compounds from $P$. ostreatus and $S$. cerevisiae intake at a dose of $0.1 \mathrm{~mL} / \mathrm{kg}$ body weight led to a decrease in ALT activity in 19 out of 20 . In one of them by $16.0 \%$ compared with the initial level, in the remaining 18 by $21.0-60.0 \%$, while in 14 patients $(70.0 \%)$ this decrease was significant, by $40.0-55.0 \%$ (Fig. 5a). It should be noted that in 11 patients $(55.0 \%)$, ALT activity reached the reference values (Fig. 5a). On average, in 19 patients, ALT activity decreased by $41.6 \%$ (Fig. 5b). And only in one of the patients (No. 3), while receiving biologically active compounds from $P$. ostreatus and $S$. cerevisiae, was an increase in ALT activity observed, by $17.8 \%$ from the initial value (marked with an arrow).

Therefore, disruption of the functional activity of liver cells, regardless of etiology, which are manifested by disruption of hepatocyte membranes and, as a consequence, the "release" of ALT into the bloodstream, can be partially prevented by 10 -day intake of biologically active compounds from $P$. ostreatus and $S$. cerevisiae at a dose of $0.1 \mathrm{~mL} / \mathrm{kg}(5 \mathrm{~mL}$ day), except for patient No. 3 .

Another indicator of the functional state of the liver is the activity of aspartate aminotransferase (AST). It was found that in three patients it corresponded to the upper reference values $(0.47 \mathrm{mmol} / \mathrm{h} * \mathrm{~mL})$, in 4 patients the activity of this enzyme was increased by $43.0 \%$ and in 13 patients an increase of 2.1 times compared with the upper limits of the reference values (Fig. 6a). In patient No. 3, AST activity was increased by $133.0 \%$ (Fig. 6a). A ten-day course of taking biologically active compounds from $P$. ostreatus and $S$. cerevisiae at a dose of $0.1 \mathrm{~mL} / \mathrm{kg}$ of body weight led to a decrease in AST activity in the blood serum, while in the majority (13 people) it decreased by $40.0-60.0 \%$ from the initial values (Fig. 6b). In 12 patients, AST activity, after a ten-day course of biologically active compounds administration, corresponded to the reference values (Fig. 6a). On average, in 19 patients, AST activity decreased by $45.6 \%$ (Fig. 6b). In patient No. 3, while receiving biologically active compounds, an increase in AST activity by $33.0 \%$ from the initial value was observed (Fig. 6a).
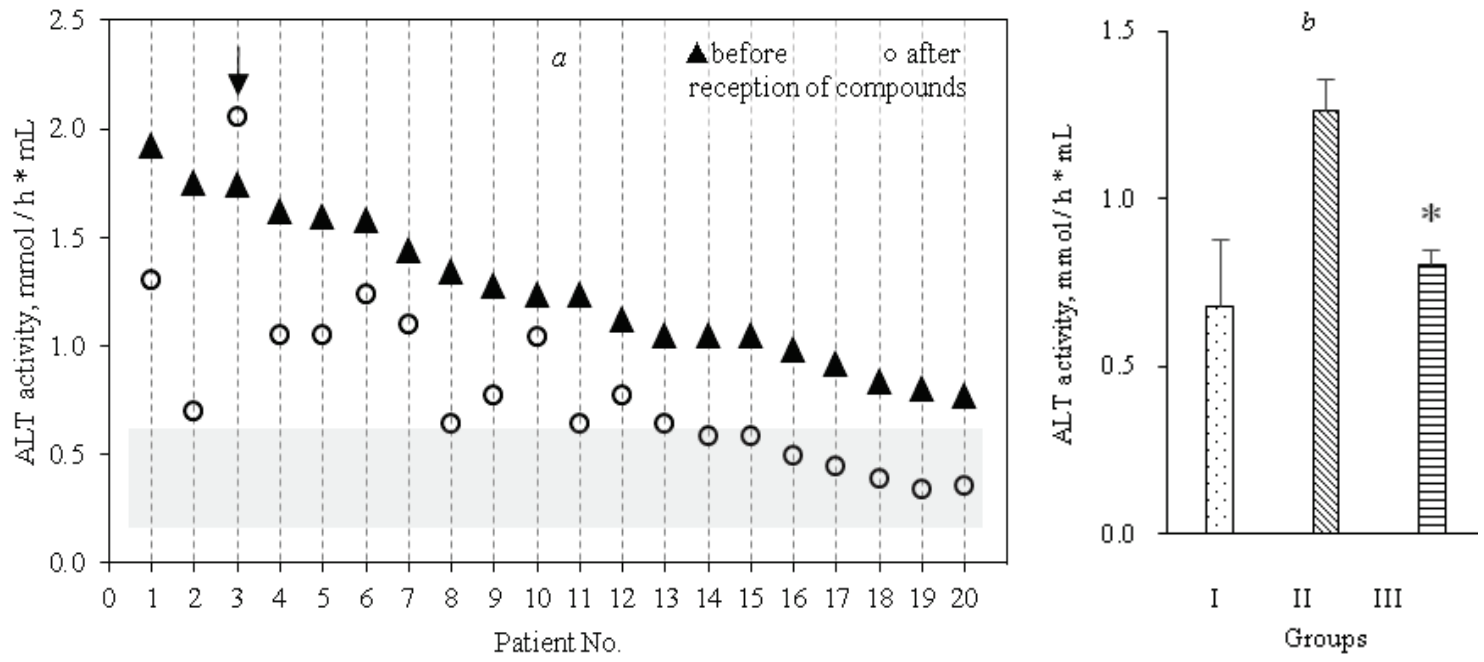

Fig. 5. ALT activity $(\mathrm{x} \pm \mathrm{SE}, \mathrm{N}=20)$ : $a$-ALT activity in blood serum in patients before taking biologically active compounds from $P$. ostreatus and S. cerevisiae $(\boldsymbol{\Delta})$ - initial level and its activity after 10-day daily intake of compounds at a dose of $0.1 \mathrm{~mL} / \mathrm{kg}$ body weight (O), reference values are marked with the grey background; $b$ - reference value (I), the mean value of ALT activity in a group of 20 patients before taking compounds (II) and 10 days after taking compounds (III); * - significant differences were noted at $\mathrm{P}<0.05$ (taking into account the Bonferroni amendment) in comparison with the value before taking compounds; patient No. 3 is highlighted with an arrow

These results confirm the conclusion that the structure of hepatocytes is restored after 10 days' intake of biologically active compounds from $P$. ostreatus and $S$. cerevisiae administration in most patients. At the same time, the restoration of hepatocyte membranes may not reflect the detoxification function of native hepatocytes. The detoxification function of the liver, which, as a rule, is inhibited in hepatitis and fibrosis, can be judged by the content of bilirubin (a product of the hemoglobin destruction) in the blood serum. It was revealed that in the studied patients, the content of total bilirubin in the blood serum was higher than the upper normal values in 8 patients (and amounted to $48.2 \mu \mathrm{mol} / \mathrm{L}$, i.e. 2.3 times higher than the reference values), in the remaining 12 patients it was $15.0 \mu \mathrm{mol} / \mathrm{L}$, i.e. fit in the upper limit of the norm (Fig. 7a). A ten-day course of taking biologically active compounds from $P$. ostreatus and $S$. cerevisiae at a dose of
$0.1 \mathrm{~mL} / \mathrm{kg}$ of body weight led to a slight decrease in the bilirubin content by $3.0-6.0 \%$ from the initial one in three patients. In the rest of the patients, its content decreased by $23.0-53.0 \%$ from the initial level, and as a result, in 16 patients it corresponded to the reference values (Fig. 7a). On average, in the study group, except for patient No. 3, the serum bilirubin content decreased by $20.3 \%$ (Fig. $7 b$ ). In patient No. 3, while receiving biologically active compounds, an increase in the bilirubin content (as well as in the case of ALT and AST) by $29.0 \%$ was observed (Fig. 7a). Consequently, biologically active compounds from $P$. ostreatus and $S$. cerevisiae normalizes, in most patients, the activity of ALT, AST, and the serum bilirubin content after 10 days of taking biologically active compounds at a dose of $0.1 \mathrm{~mL} / \mathrm{kg}$, and the normalization effect has individual characteristics. 

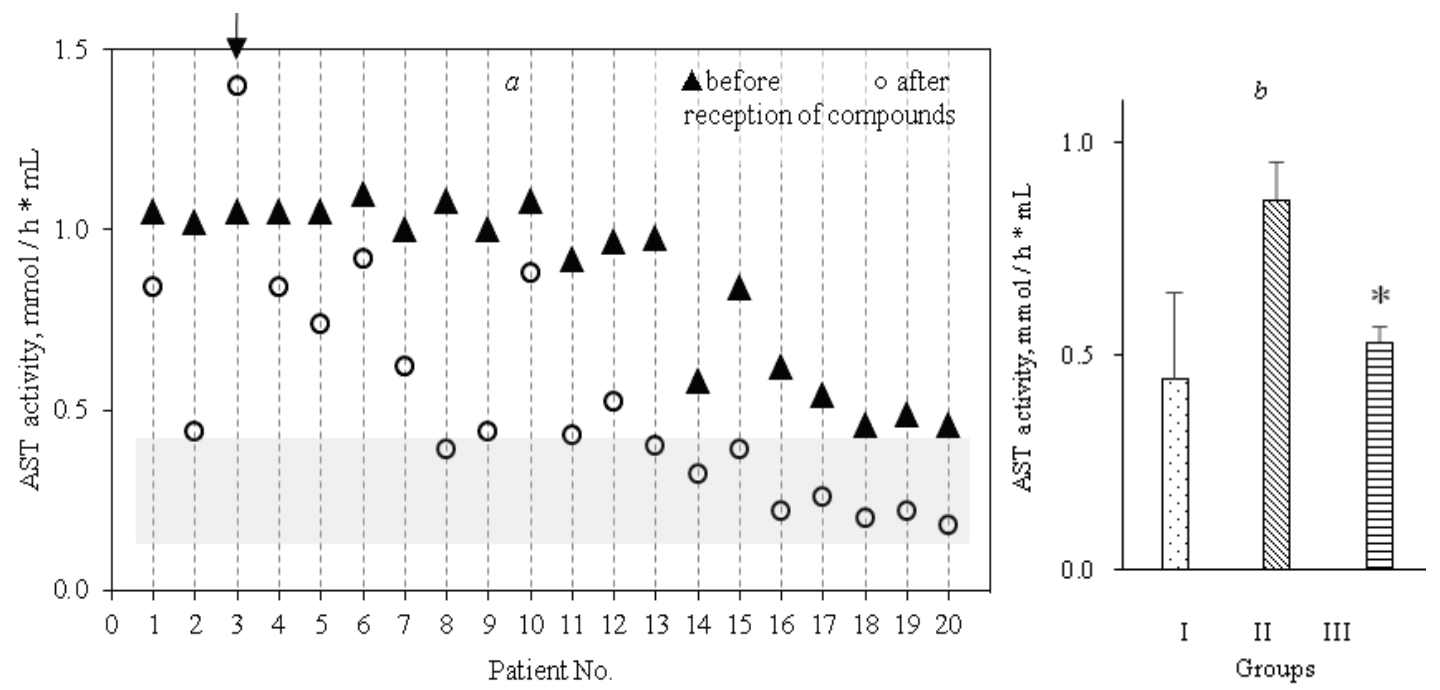

Fig. 6. AST activity $(\mathrm{x} \pm \mathrm{SE}, \mathrm{N}=20): a$ - AST activity in blood serum in 20 patients before taking biologically active compounds from $P$. ostreatus and S. cerevisiae $(\boldsymbol{\Delta})$; AST activity after 10 days of taking compounds at a dose of $0.1 \mathrm{~mL} / \mathrm{kg}$ of body weight $(\circ)$; $b$-reference value (I), mean value for the AST activity group before taking compounds (II) and 10 days after taking compounds (III); ${ }^{*}$ - significant differences were noted at $p<0.05$ (taking into account the Bonferroni amendment) in comparison with the value before taking compounds; patient No. 3 is highlighted with an arrow
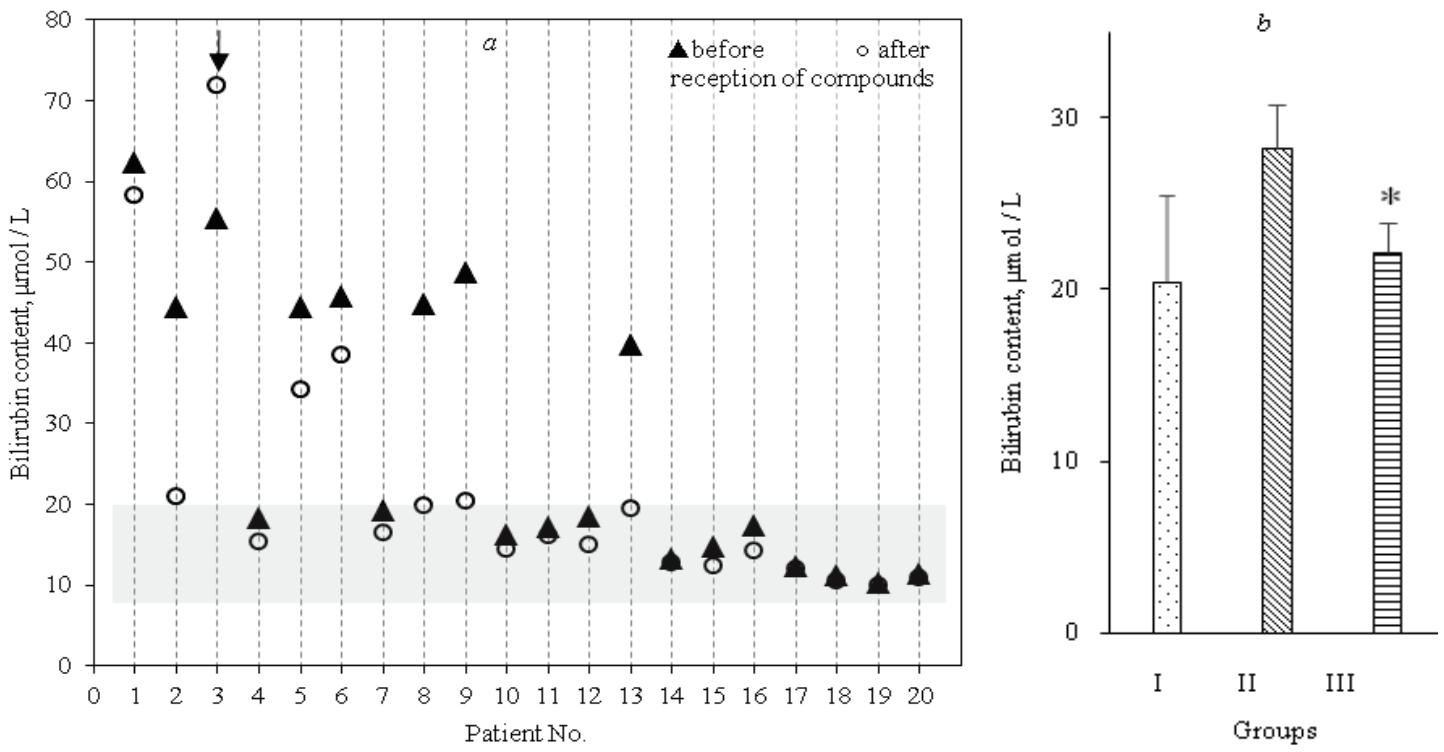

Fig. 7. The content of bilirubin ( $\mathrm{x} \pm \mathrm{SE}, \mathrm{N}=20)$ : $a$ - the content of bilirubin in blood serum in 20 patients before taking biologically active compounds from $P$. ostreatus and $S$. cerevisiae $(\boldsymbol{\Delta})$ and after 10 days of taking compounds at a dose of $0.1 \mathrm{~mL} / \mathrm{kg}$ of body weight (o); $b-$ reference value (I), the average bilirubin value in the group before taking compounds (II) and after taking compounds (III); ${ }^{*}$ - significant differences were noted at $\mathrm{P}<0.05$ (taking into account the Bonferroni amendment) in comparison with the value before taking compounds; patient No. 3 is highlighted with an arrow

\section{Discussion}

The present study, carried out on experimental animals, showed that biologically active compounds from $P$. ostreatus and S. cerevisiae components can prevent the death of most animals in the study group from acute poisoning with copper sulfate, and this is realized through the regulation of the redox system and membrane-stabilizing action, i.e. biologically active compounds are a biological antidote, and in clinical studies on volunteers, membranotropic and, as a consequence, hepatoprotective effects have been confirmed.

The results obtained allow us to assert that biologically active compounds from $P$. ostreatus and $S$. cerevisiae have a broad (multifunctional) nature of their action on biological systems, and on the other hand, the manifestation of their actions depends on the individual characteristics of the organism.

Thus, in one of 20 patients, biologically active compounds from $P$. ostreatus and S. cerevisiae did not stop the pathogenesis process, and 20 percent of the animals with $\mathrm{Cu}$-induced intoxication died, as in the control variant, i.e. biologically active compounds did not exert a protec- tive effect on them. This indicates that the action of biologically active compounds depends on the functional states of the organism, which are not defined in this case, and have an individual character.

These results raise several important questions, the search for answers to which may be important for understanding the mechanisms of action of other biologically active compounds: 1 - why biologically active compounds from $P$. ostreatus and S. cerevisiae did not have, or had an insignificant effect on a healthy organism and were active in the presence of pathologies; 2 - why, in some cases, biologically active compounds from $P$. ostreatus and $S$. cerevisiae did not affect the pathologically altered state, i.e. did not show an "induced" protective response; 3 - what explains the U-shaped dose dependence of the biologically active compounds on the action of $P$. ostreatus and S. cerevisiae; 4 - how to explain the multifunctional effect of biologically active compounds from $P$. ostreatus and S. cerevisiae on the body.

It can be assumed that if the organism is "healthy", which means that its metabolic system is in a relatively stable homeostatic state, it is capable of self-maintenance and therefore external factors, if they do not exert a "strong" disturbance, will not affect the metabolic activity. Since the bio- 
logically active compounds from $P$. ostreatus and $S$. cerevisiae components are of biological origin, i.e. are not xenobiotics and are not capable of exerting a "strong" effect on the metabolically stable system, but are capable of exerting regulatory "soft" effects, then such a system is not sensitive to their action.

Since biologically active compounds from $P$. ostreatus and S. cerevisiae enter the body per os, its components can perform trophic functions. The possible trophic role of biologically active compounds is supported by data on a slight increase in body weight and performance in control animals (Kurguzova et al., 2015; Bozhkov et al., 2016).

It can be assumed that the metabolic system of a "healthy" organism can maintain itself in an "equilibrium" state and the biologically active compounds from $P$. ostreatus and S. cerevisiae components are not able to shift its equilibrium. In this case, biologically active compounds will exhibit only trophic functions.

If a pathological process takes place in the body, i.e. the homeostatic state is disturbed and the metabolic system of the organism passes into a "new functional state", which can be characterized as far from equilibrium (Ebrahimi et al., 2016), then the biologically active compounds from $P$. ostreatus and S. cerevisiae components, along with trophic ones, also exhibit various regulatory effects.

At the initial stages of the development of pathology, the metabolic system is "able" to restore its homeostatic state; this state can be defined as reversible. At the later stages of the development of the pathological process, the system passes into irreversible states, i.e. the system becomes homeostatically stable again. This condition is, in practice, defined as "irreversible" or chronic. In the case of chronic condition, i.e. a new steady-state, biologically active compounds from $P$. ostreatus and $S$. cerevisiae will also not have a significant regulatory effect, which may have taken place in patient No. 3 and some of the animals that died after the administration of copper sulfate large doses.

Consequently, in the case of a stable metabolic state (a healthy organism or a chronic pathological state), biologically active compounds from $P$. ostreatus and S. cerevisiae will not have any effect, while in the case of a metabolic disequilibrium state, the biologically active compounds will contribute to the restoration of homeostatic equilibrium.

Lackey wrote about this figuratively in his time: "A pathological organism responds to the impact with ease if it is in suboptimal conditions, that is, the metabolic system is quasi-stable and far from the state of homeostatic equilibrium, it reacts faster than a system in relative equilibrium". Such states are very well described for physical systems (Thibault et al., 2012).

Based on this position, it can be assumed that the actions of the biologically active compounds from $P$. ostreatus and $S$. cerevisiae components depend on the state of the metabolic system at the time of exposure. If the system is in homeostatic equilibrium or close to it, a healthy organism or an organism with chronic pathology (which has become a new stable state), then such a system retains its previous state, and biologically active compounds from $P$. ostreatus and $S$. cerevisiae will not influence it.

Of course, these general explanations require deep detailing, but this allows us to understand the direction of further search for the mechanism of biologically active compounds from $P$. ostreatus and $S$. cerevisiae action. If these judgments are correct, then biologically active compounds can be used to determine the state of the metabolic system in relation to homeostatic equilibrium, which can be important in comparative studies.

Establishing the dose dependence of the body's response to an external factor is important in understanding the mechanisms of action of biological substances. As has been shown in experimental animals, the socalled U-shaped dose dependence is characteristic of the biologically active compounds from $P$. ostreatus and $S$. cerevisiae, i.e. there is a maximum response, at conditionally average concentrations of substances, while small and large doses do not induce a recorded response.

As is known, this dependence reflects the phenomenon of hormesis nonspecific stimulation of biological processes by small doses of factors harmful to the body, in which the toxic effect does not manifest itself (Kendig et al., 2010). Hormesis is shown at low doses of radiation (radiation hormesis) (Hickey et al., 1983), toxic compounds, in particular copper sulfate (Kovaleva et al., 2012; Bozhkov et al., 2014), and is a general biological phenomenon (Calabrese \& Baldwin, 1998).
However, unlike toxic compounds, biologically active compounds from $P$. ostreatus and $S$. cerevisiae do not show negative, depressing effects, even at high doses $(0.8 \mathrm{~mL} / 100 \mathrm{~g}$ of body weight). In this respect, this substance cannot be claimed to exert hormesis, and the analogy with the hormesis effect can be defined as a quasi-hormesis effect.

The quasi-hormesis effect of biologically active compounds from $P$. ostreatus and S. cerevisiae can be explained by the principle of functional request. Since the biologically active compounds consists of a large number of various biologically active components (Fig. 2), which of these substances will exhibit one or another activity depends on which of the endogenous ligands of the metabolic system is capable of interacting with biologically active compounds at a given time.

An example is the functioning of the body's redox system. It has been shown that the same biological components, depending on the state, can perform the functions of both prooxidants and antioxidants. It was previously shown that the components of cow colostrum, depending on the dose, can perform the functions of both an antioxidant and a prooxidant (Bozhkov et al., 2017).

As it known, most of the known enzymes are polyfunctional, and which of its activities the enzyme will exhibit depends on the functional request of the system, which is aimed at maintaining homeostatic equilibrium at a given time.

It should be noted that the polyfunctionality that was fixed at the physiological level is formed in different ways at the levels of the hierarchical organization of biological systems. The action of biologically active compounds from $P$. ostreatus and S. cerevisiae can be "direct" - this is when the components of a multicomponent substance directly interact with certain ligands, i.e., they perform a regulatory role based on the principle of functional request. Along with this, biologically active compounds from $P$. ostreatus and $S$. cerevisiae can also have an indirect effect on the functional characteristics of biosystems. Thus, in the case of a healthy organism, biologically active compounds from $P$. ostreatus and $S$. cerevisiae can perform a trophic function and have an indirect effect on manybody systems. So, the administration of biologically active compounds from $P$. ostreatus and $S$. cerevisiae to animals against the background of bacterial infection (P. aeruginosa and E. coli) has an immunotropic effect on the body (Bozhkov et al., 2017; Klimova et al., 2018).

It can be assumed that changes in the characteristics of such regulatory systems of the body as the redox system, the immune system, the digestive system, and biologically active compounds from $P$. ostreatus and S. cerevisiae can cause a synergistic effect.

As is known, synergy is joint action in one direction of some substances that make up the substrate, which provides not just summation, but emergence (i.e., it registers an effect that is more than just the sum of effects) (Kesic, 2016). It can be assumed that biologically active compounds from $P$. ostreatus and S. cerevisiae exhibit "emergent synergism" if the metabolic elements are in a state far from homeostatic equilibrium.

If we accept these assumptions and analyze the data obtained, we can put forward a working hypothesis about the "emergent-synergistic" effect of biologically active compounds from $P$. ostreatus and $S$. cerevisiae on metabolic systems that are far from homeostatic equilibrium, in particular, caused by intoxication of the body, which is accompanied by the formation of liver pathology.

Biologically active compounds from $P$. ostreatus and $S$. cerevisiae components, entering the digestive tract, affect the intestinal microflora, and, depending on the dose, they shift the equilibrium between the types of microflora, acting as a stimulant for some species and as a growth inhibitor for other species, that is, they perform a selective function (Fig. 8).

Taking into account the diverse composition of biologically active compounds from $P$. ostreatus and $S$. cerevisiae, the influence of some components on the functional activity of epithelial and secretory cells of the digestive tract cannot be ruled out. Some of the low molecular weight components undergo hydrolysis and perform trophic functions. Low molecular weight biologically active compounds from $P$. ostreatus and S. cerevisiae components entering the bloodstream can shift the balance of prooxidant activity towards antioxidants (Klimova et al., 2018). And, as a consequence, to exert both direct and indirect influence on the activity of the immune system, along with this, they also perform membranotropic functions. 


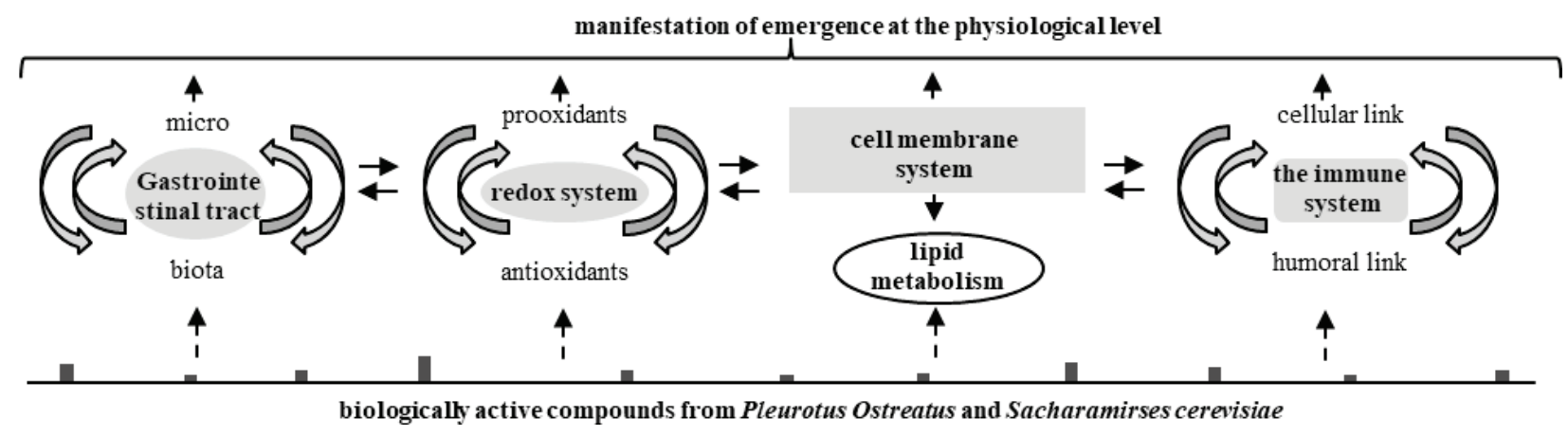

Fig. 8. Diagram showing the direct $(\rightarrow)$ and indirect $(\rightarrow)$ effects of various components of low molecular weight complexes on various functional systems (gastrointestinal tract; redox system; cell membrane system; immune system) of the body

\section{Conclusion}

The present study, carried out on experimental animals, showed that biologically active compounds from $P$. ostreatus and $S$. cerevisiae components can prevent the death of most animals in the study group from acute poisoning with copper sulfate, and this is realized through the regulation of the redox system and membrane-stabilizing action, i.e. biologically active compounds from $P$. ostreatus and $S$. cerevisiae are a biological antidote, and in clinical studies on volunteers, membranotropic and, as a consequence, hepatoprotective effects have been confirmed. The results obtained allow us to assert that biologically active compounds from $P$. ostreatus and S. cerevisiae have a broad (multifunctional) nature of action on biological systems, and on the other hand, the manifestation of their actions depends on the individual characteristics of the organism.

The authors state that there are no conflicts of interest. There are no persons who have a financial interest in the received data.

\section{References}

Asakawa, T., \& Matsushita, S. (1980). Coloring conditions of thiobarbituric acid test for detecting lipid hydroperoxides. Lipids, 15(3), 137-140.

Bozhkov, A. I., Ivanov, E. G., Al Begai, M. A., Alsardia, M. M., \& Kurguzova, N. I. (2017). Low-molecular weight cow colostrum components in functional nutrition. Journal of Nutritional Therapeutics, 6(1), 11-17.

Bozhkov, A. I., Klimova, E. M., Nikitchenko, Y. V., Davydov, V. V., Zvyagintseva, O. V., Kurguzova, N. I., \& Naglov, A. V. (2014). Stem cells take part in regulation of prooxidant activity and immunity at liver fibrosis. American Journal of Biomedical and Life Sciences, 2, 5-12.

Bozhkov, A. I., Nikitchenko, Y. V., Klimova, E. M., Linkevych, O. S., Lebid, K. M., Al-Bahadli, A. M. M., \& Alsardia, M. M. A. (2017). Young and old rats have different strategies of metabolic adaptation to $\mathrm{Cu}$-induced liver fibrosis. Advances in Gerontology, 7(1), 41-50.

Bozhkov, A. I., Nikitchenko, Y. V., Lebed, K. N., Linkevych, O. S., Kurguzova, N. I., Klimova, O. M., Begai A. Y., Al-Bahadly A. M., \& Alsardia, M. M. (2016). The cyclic feeding regime induces decaying age-dependent oxidative stress and regulates the cell chain of the immunity. Advances in Aging Research, 5(6), 151-165.

Bozhkov, A. I., Padalko, V., Dlubovskaya, V., \& Menzianova, N. (2010). Resistance to heavy metal toxicity in organisms under chronic exposure. Indian Journal of Experimental Biology, 48(7), 679-696.

Bozhkov, A. I., Sidorov, V. I., Kurguzova, N. I., \& Dlubovskaia, V. L. (2014). Metabolic memory enhances hormesis effect to the copper ions in age-depended manner. Advances in Gerontology, 27(1), 72-80.

Buchanan, R., \& Sinclair, J. M. (2021). Alcohol use disorder and the liver. Addiction, $116(5), 1270-1278$.

Calabrese, E. J., \& Baldwin, L. A. (1998). Hormesis as a biological hypothesis. Environmental Health Perspectives, 106(1), 357-362.

Ebrahimi, H., Naderian, M., \& Sohrabpour, A. A. (2016). New concepts on pathogenesis and diagnosis of liver fibrosis: A review article. Middle East Journal of Digestive Diseases, 8(3), 166.

Fahmy, M. A., Hassan, E. E., Ibrahim, N. E., Hassan, E. M., Hassan, Z. M., \& Omara, E. A. (2020). Protective role of ficus carica extract against hepato-testicular side effects and genotoxicity induced by cisplatin. Pharmacognosy Journal, $12(3), 645-656$.
Francavilla, A., Hagiya, M., Porter, K. A., Polimeno, L., Ihara, I., \& Starzl, T. E. (1994). Augmenter of liver regeneration: Its place in the universe of hepatic growth factors. Hepatology, 20(3), 747-757.

Gagliano, N., Grizzi, F., \& Annoni, G. (2007). Mechanisms of aging and liver functions. Digestive Diseases, 25(2), 118-123.

Gao, B., \& Bataller, R. (2011). Alcoholic liver disease: Pathogenesis and new therapeutic targets. Gastroenterology, 141(5), 1572-1585.

Hickey, R. J., Bowers, E. J., \& Clelland, R. C. (1983). Radiation hormesis, public health, and public policy: A commentary. Health Physics, 44(3), 207-219.

Hirsova, P., Ibrahim, S. H., Verma, V. K., Morton, L. A., Shah, V. H., LaRusso, N. F., Gores, G. J., \& Malhi, H. (2016). Extracellular vesicles in liver pathobiology: Small particles with big impact. Hepatology, 64(6), 2219-2233.

Jaramillo-Juárez, F., Rodríguez-Vázquez, M. L., Rincón-Sánchez, A. R., Martínez, M. C., Ortiz, G. G., Llamas, J., Posadas, F. A., \& Reyes, J. L. (2008). Acute renal failure induced by carbon tetrachloride in rats with hepatic cirrhosis. Annals of Hepatology, 7(4), 331-338.

Kamath, S. A., \& Narayan, K. A. (1972). Interaction of $\mathrm{Ca}^{2+}$ with endoplasmic reticulum of rat liver: A standardized procedure for the isolation of rat liver microsomes. Analytical Biochemistry, 48(1), 53-61.

Kendig, E. L., Le, H. H., \& Belcher, S. M. (2010). Defining hormesis: Evaluation of a complex concentration response phenomenon. International Journal of Toxicology, 29(3), 235-246.

Kesić, S. (2016). Systems biology, emergence and antireductionism. Saudi Journal of Biological Sciences, 23(5), 584-591.

Klimova, E. M., Bozhkov, A. I., Kovalenko, T. I., Minukhin, V. V., \& Belozerov, I. V. (2018). Young and old animals use different strategies for forming an immune response to infectious agents (Pseudomonas aeruginosa and Escherichia coli). Advances in Gerontology, 8(4), 284-291.

Kovaleva, M. K., Menzyanova, N. G., Jain, A., Yadav, A., Flora, S., \& Bozhkov, A. I. (2012). Effect of hormesis in Dunaliella viridis Teodor. (Chlorophyta) under the influence of copper sulfate. International Journal on Algae, 14(1), 41-61.

Kurguzova, N. I., Bozhkov, A. I., Nikitchenko, Y. V., Al Begai, M. A. Y., Goltvyansky, A. V., Alsardia, M. M. A., \& Bozhkov, A. A. (2015). Interconnection of antitoxic and antioxidant systems of the organism under the action of natural low molecular complex - fungidol. American Journal of Biomedical and Life Sciences, 2, 25-32.

Lakherwal, D. (2014). Adsorption of heavy metals: A review. International Journal of Environmental Research and Development, 4(1), 41-48.

Lynes, M. A., Kang, Y. J., Sensi, S. L., Perdrizet, G. A., \& Hightower, L. E. (2007). Heavy metal ions in normal physiology, toxic stress, and cytoprotection. Annals of New York Academy of Sciences, 1113, 159.

Mann, H. B., \& Whitney, D. R. (1947). On a test of whether one of two random variables is stochastically larger than the other. The Annals of Mathematical Statistics, 18(1), 50-60.

Mason, C., \& Manzotti, E. (2010). Regenerative medicine cell therapies: Numbers of units manufactured and patients treated between 1988 and 2010. Regenerative Medicine, 5(3), 307-313.

Michalopoulos, G. K., \& DeFrances, M. C. (1997). Liver-regeneration. Science, 277(5331), 1423-1423.

Myers, R. P., Ratziu, V., Benhamou, Y., Di Martino, V., Moussalli, J., Hélène Tainturier, M., \& Poynard, T. (2002). Infections with multiple hepatotropic viruses. In: Brogden, K. A., \& Guthmiller, J. M. (Eds.). Polymicrobial Diseases. Wiley. Pp. 51-73.

Owojuyigbe, O. S., Firempong, C. K., Larbie, C., Komlaga, G., \& Emikpe, B. O. (2020). Hepatoprotective potential of Hura crepitans L.: A review of ethnomedical, phytochemical and pharmacological studies. Journal of Complementary and Alternative Medical Research, 9(2), 1-10.

Paglia, D. E., \& Valentine, W. N. (1967). Studies on the quantitative and qualitative characterization of erythrocyte glutathione peroxidase. The Journal of Laboratory and Clinical Medicine, 70(1), 158-169. 
Parkinson, A., \& Ogilvie, B. W. (2008). Biotransformation of xenobiotics. Casarett and Doull's Toxicology: The Basic Science of Poisons, 7, 161-304.

Piven, O. T., Khimych, M. S., Salata, V. Z., Gutyj, B. V., Naidich, O. V., Skrypka, H. A., Koreneva, Z. B., Dvylyuk, I. V., Gorobey, O. M., \& Rud, V. O. (2020). Contamination of heavy metals and radionuclides in the honey with different production origin. Ukrainian Journal of Ecology, 10(2), 405-409.

Poordad, F. F. (2015). Presentation and complications associated with cirrhosis of the liver. Current Medical Research and Opinion, 31(5), 925-937.

Puche, J. E., Saiman, Y., \& Friedman, S. L. (2011). Hepatic stellate cells and liver fibrosis. Comprehensive Physiology, 3(4), 1473-1492.

Rana, A., \& Godfrey, E. L. (2019). Outcomes in solid-organ transplantation: Success and stagnation. Texas Heart Institute Journal, 46(1), 75-76.

Saito, Z., Kaneko, Y., Kinoshita, A., Kurita, Y., Odashima, K., Horikiri, T., \& Kuwano, K. (2016). Effectiveness of hepatoprotective drugs for anti-tuberculosis drug-induced hepatotoxicity: A retrospective analysis. BMC Infectious Diseases, 16(1), 1-6.

Satapathy, S. K., Kuwajima, V., Nadelson, J., Atiq, O., \& Sanyal, A. J. (2015). Druginduced fatty liver disease: An overview of pathogenesis and management. Annals of Hepatology, 14(6), 789-806. http://doi.org/10.5604/16652681.1171749

Sepanlou, S. G., Safiri, S., Bisignano, C., Ikuta, K. S., Merat, S., Saberifiroozi, M. (2020). The global, regional, and national burden of cirrhosis by cause in 195 countries and territories, 1990-2017: A systematic analysis for the Global Burden of Disease Study 2017. The Lancet Gastroenterology and Hepatology, 5(3), 245-266.
Shirani, M., Raeisi, R., Heidari-Soureshjani, S., Asadi-Samani, M., \& Luther, T. (2017). A review for discovering hepatoprotective herbal drugs with least side effects on kidney. Journal of Nephropharmacology, 6(2), 38-48.

Slivinska, L. G., Vlizlo, V. V., Shcherbatyy, A. R., Lukashchuk, B. O., Gutyj, B. V., Drach, M. P., Lychuk, M. G., Maksymovych, I. A., Leno, M. I., Rusyn, V. I., Chernushkin, B. O., Fedorovych, V. L., Zinko, H. O., Prystupa, O. I., \& Yaremchuk, V. Y. (2021). Influence of heavy metals on metabolic processes in cows. Ukrainian Journal of Ecology, 11(2), 284-291.

Thibault, G., Shui, G., Kim, W., McAlister, G. C., Ismail, N., Gygi, S. P., Wenk, M. R., \& Ng, D. T. (2012). The membrane stress response buffers lethal effects of lipid disequilibrium by reprogramming the protein homeostasis network. Molecular Cell, 48(1), 16-27.

Varghese, S., Tang, Y., \& Imlay, J. A. (2003). Contrasting sensitivities of Escherichia coli aconitases A and B to oxidation and iron depletion. Journal of Bacteriology, 185(1), 221-230.

Verma, V. K., Sarwa, K. K., Kumar, A., \& Zaman, M. K. (2013). Comparison of hepatoprotective activity of Swertia chirayita and Andrographis paniculata plant of North-East India against $\mathrm{CCl}_{4}$ induced hepatotoxic rats. Journal of Pharmacy Research, 7(7), 647-653.

Williams, G. M., \& Iatropoulos, M. J. (2002). Alteration of liver cell function and proliferation: Differentiation between adaptation and toxicity. Toxicologic Pathology, 30(1), 41-53.

Xu, G. B., Xiao, Y. H., Zhang, Q. Y., Zhou, M., \& Liao, S. G. (2018). Hepatoprotective natural triterpenoids. European Journal of Medicinal Chemistry, 145, 691-716. 